BULL. AUSTRAL. MATH. SOC.

VOL. I (.1969), 279-280.

\title{
The completion by cuts of an orthocomplemented modular lattice
}

\author{
D. H. Adams
}

In this note we give an example of an orthocomplemented modular lattice whose completion by cuts is not orthomodular. This solves negatively Problem 36, p. 131, in G. Birkhoff: Lattice theory (3rả edition).

Let $V$ be an infinite dimensional prehilbert space over the complex field $C$. Assume that $V$ is incomplete with respect to the inner product $(\cdot, \cdot)$. Let $L(V)$ be the lattice of subsets of $V$ closed under the closure operation $S \rightarrow S^{\perp \perp}$ where

$$
S^{\perp}=\{f: f \in V,(f, g)=0, \forall g \in S\} .
$$

It is easy to see that the elements of $L(V)$ are linear subspaces of $V$, and it follows from the lemma on $p .425$ of [1] that all finite dimensional subspaces of $V$ belong to $L(V)$. It is straightforward to show that the map $\perp: L(V) \rightarrow L(V)$ is an orthocomplementation in $L(V)$ ([2], p. 123).

Now let $L_{1}(V)$ be the sublattice of $L(V)$ consisting of all finite dimensional subspaces of $V$ and their orthocomplements. Then $L_{l}(V)$ is an orthocomplemented modular lattice, and it is also join-dense in $L(V)$. It follows from a theorem of $M$. Donald MacLaren that the completion by cuts of $L_{1}(V)$ is isomorphic to the complete lattice $L(V)$ ([3], Th. 2.5). As $V$ was assumed to be incomplete, $L(V)$ is not orthomodular by the main theorem in [1].

Received 23 March 1969. 
We must now show the existence of such an incomplete prehilbert space $V$. The space of all continuous, absolutely square integrable functions on the closed interval $[0,1]$ is such a space with the usual $L_{2}$ inner product. So the lattice $L_{l}(V)$ of finite and cofinite dimensional subspaces of $V$ is the promised example.

\section{References}

[1] Ichiro Amemiya and Huzikiro Araki, "A remark on Piron's paper", Pubz. Res. Inst. Math. Sci. Ser. A, Kyoto, 2 (1967), pp. 423 427.

[2] G. Birkhoff, Lattice Theory, 3rd. ed. Amer. Math. Soc. Colloquium Publications Vol. 25, (Amer. Math. Soc., Providence, R.I., 1967).

[3] M. Donald MacLaren, "Atomic orthocomplemented lattices", Pacific J. Math. 14 (1964), pp. 597-612.

Monash University,

Clayton, Victoria, Australia. 